\title{
Comparison of Impurities and Time-Dependent Behavior for the ITER Divertor
}

\author{
M.E. Rensink, T.D. Rognlien, and D.D. Hua
}

February 25, 1997

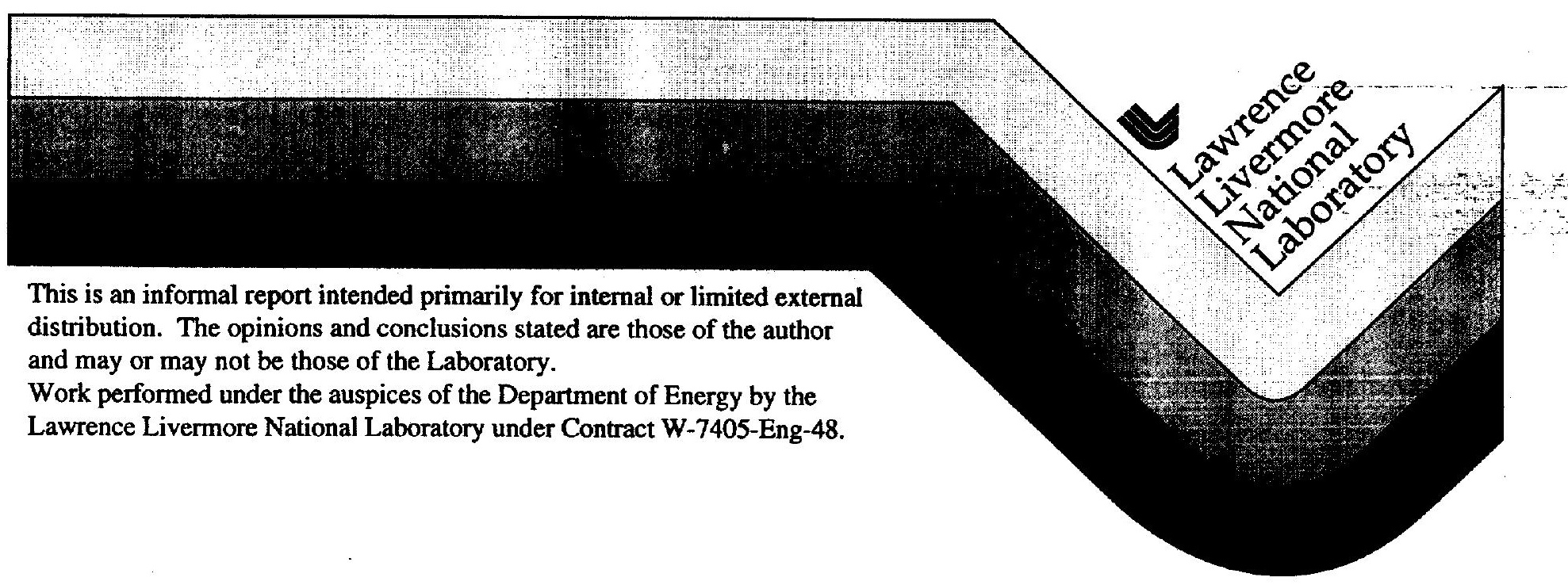




\section{DISCLAIMER}

This document was prepared as an account of work sponsored by an agency of the United States Government. Neither the United States Government nor the University of California nor any of their employees, makes any warranty, express or implied, or assumes any legal liability or responsibility for the accuracy, completeness, or usefulness of any information, apparatus, product, or process disclosed, or represents that its use would not infringe privately owned rights. Reference herein to any specific commercial product, process, or service by trade name, trademark, manufacturer, or otherwise, does not necessarily constitute or imply its endorsement, recommendation, or favoring by the United States Government or the University of California. The views and opinions of authors expressed herein do not necessarily state or reflect those of the United States Government or the University of California, and shall not be used for advertising or product endorsement purposes.

This report has been reproduced directly from the best available copy.

Available to DOE and DOE contractors from the Office of Scientific and Technical Information

P.O. Box 62, Oak Ridge, TN 37831

Prices available from (615) 576-8401, FTS 626-8401

Available to the public from the

National Technical Information Service

U.S. Department of Commerce

5285 Port Royal Rd.

Springfield, VA 22161 


\title{
Comparison of Impurities and Time-Dependent Behavior for the ITER Divertor
}

\author{
M.E. Rensink, T.D. Rognlien, and D.D. Hua \\ Lawrence Livermore National Laboratory \\ Livermore, CA 94551
}

This a the second part-of an ongoing project to model the divertor plasma for ITER. The UEDGE 2-D edge transport code is used to study the effect of impurities and tilted divertor plates to make-a-fadiative divertor that can prevent extessive teat toads and adequately pump helium produced by fusion reactions in the core. The impurities are modeled using individual charge states with the local concentrations being determined by transport or as a fixed fraction of the hydrogenic ion density. For the multi-species model, helium, beryllium, carbon, and neon impurities are considered separately, together with the majority hydrogenic species, and a comparison is made of impurity spatial distribution and the power radiated at low impurity levels. At moderate to high impurity levels, typically only time-dependent solutions are found which are studied here for neon using both impurity models. 


\section{Contents}

I Introduction $\quad 2$

II Comparison of Four Impurities $\quad \ldots \quad 4$

A Background Hydrogen Features ................... 4

B Spatial Distribution of Impurity Radiation . . . . . . . . . . . 5

C Solutions with Strong Sputtering $\ldots \ldots \ldots \ldots$

D Inter-species Charge Exchange $\ldots \ldots \ldots \ldots$

IIIBehavior as Detached Solution Is Approached and Comparison of Impurity Models

A Oscillatory.Solutions:

B Slowhy-Evolving Detached Solutions $\ldots \ldots \ldots \ldots \ldots$

\section{Introduction}

This status report presents results obtained for modeling the ITER divertor using the UEDGE code for the time period July-Dec, 1996. The basic models used in the code and our first set of results for the ITER EDA are described in our first status report, Ref. 1. We assume the reader is familiar with contents of the first report and here focus on the new results.

First, we give a brief description of the content of the 2-D edge/SOL transport code..$^{3-7}$ UEDGE solves the classical Braginskii ${ }^{2}$ transport equations for plasma transport along the magnetic field, $\mathbf{B}$, and assumes anomalous diffusive transport across $\mathbf{B}$. The neutral gas that recycles from the divertor plate is modeled by fluid equations where inertia is retained in 
the parallel direction along $\mathbf{B} .^{8}$ In comparisons with a more complete Navier-Stokes neutral gas model in a slab version of UEDGE, ${ }^{9}$ this reduced Navier-Stokes model has been shown to accurately represent the ion-neutral momentum exchange and the collisionally enhanced momentum and energy transport including neutral-neutral collisions. The nonorthogonal mesh algorithm in UEDGE makes use of a general 9-point difference stencil which preserves the magnetic flux surfaces as one coordinate to resolve the highly anisotropic transport along and across $\mathbf{B} .^{6}$ The parallel transport of the multi-charge state impurities is described by force-balance equations ${ }^{6}$ using the trace-level coefficients presented earlier ${ }^{10,11}$ or by the FMOMBAL package. ${ }^{12}$ Atomic data for hydrogen rates of excitation, ionization, and recombination by electrons is taken from the work of Stotler, ${ }^{13}$ and the impurity rates are from STRAHL ${ }^{14}$ via a B2.5 package by Braams. ${ }^{15}$

Our previous studies ${ }^{1}$ of helium and neon for orthogonal and tilted divertor plates has been extended to the intrinsic impurities beryllium and carbon. In Sec. II, a comparison is given of the spatial density distribution and radiation for each of the four impurity species. These results are for the outer-half of the scrape-off layer (SOL) and include steady-state solutions for attached and partially detached divertor plasmas.

As discussed in the first report, ${ }^{1}$ as the impurity level is increased beyond a certain value, we were unable to obtain steady-state solutions. In Sec. III, a detailed examination is made of the impurity-behavior with neon as-this steady-state solution boundary is approached. Results of the fixed-fraction and multi-charge-state impurity models are compared. In Sec. IV, we examine this loss of steady-state solutions in some detail and show that two types of time-dependent solutions appear. The first arises if the core boundary condition is one of constant density, and then the position of the detached ionization front slowly moves upstream. This slow movement occurs at a rate at which the finite plasma flux across the core boundary provides a particle source to build the neutral density in the volume between the ionization front and the divertor plate to densities in the range of $\sim 3 \times 10^{21} \mathrm{~m}^{-3}$. This is typically hundreds of msec. The second type of solution occurs if the core density boundary condition is zero flux. Then oscillatory solutions are obtained that can change between weakly attached and detached. A MARFE just under the $x$-point participates in this oscillation, although whether the MARFE is helping drive the variations or just being driven is yet to be determined. These oscillations are likely the same type as those discussed 
in Ref. 16.

\section{Comparison of Four Impurities}

We compare the behavior of $\mathrm{He}, \mathrm{Be}, \mathrm{C}$ and $\mathrm{Ne}$ impurities for a half-space configuration with orthogonal and moderately tilted divertor plate. For each impurity, we specify a fixed ion density (usually, that of the fully-stripped charge state) at the core boundary. Zeroparticle-flux boundary conditions are imposed at the sidewalls for both ionized and neutral impurities.

For recycling impurities, He and Ne, the impurity gas flux from the divertor plate is just equal to the total impurity ion flux incident on the plate. There is no net source or sink of particles in the system. The impurity concentration in the scrape-off layer is controlled by the core boundary condition.

For non-recycling impurities, Be and $\mathrm{C}$, we simulate physical sputtering by setting the the-impurity gas flux from the divertor plate equal to some fraction of the hydrogenic ion flux incident on the plate: The constant of proportionality is the sputtering yield, $Y_{\text {phys }}$, which is independent of temperature in our model. This impurity particle source due to physical sputtering is partially pumped at the divertor plate because ionized impurities incident on the plate do not recycle (no self-sputtering); the remainder of the impurities may be convected upstream to the core region (by friction on hydrogenic reversed flows or by the ion thermal force) where they accumulate until their density exceeds the fixed-density boundary value and they diffuse radially out of the scrape-off layer into the core region. In this situation, the impurity concentration in the scrape-off layer is controlled by both the sputtering yield and the core boundary condition.

\section{A. Background Hydrogen Features}

Input parameters for the hydrogenic plasma are the core plasma density, $\mathrm{n}_{\text {core }}=3 \times 10^{19} \mathrm{~m}^{-3}$, and the power flow into the outer half of the scrape-off layer, $P_{\text {core }}=100 \mathrm{MW}$ (equally split between electrons and ions). The anomalous diffusion coefficients correspond to the so-called weak diffusion case ${ }^{1}$ with $D_{\perp}=0.33 \mathrm{~m}^{2} / \mathrm{s}$ and $\chi_{e, i}=0.5 \mathrm{~m}^{2} / \mathrm{s}$. For an orthogonal divertor 
plate, this produces an attached plasma with a peak plate electron temperature of $26 \mathrm{eV}$ and a peak plate heat flux of $75 \mathrm{MW} \mathrm{m}^{-2}$. The total radiated power is $5.6 \mathrm{MW}$, mostly due to ionization within a few centimeters of the divertor plate. Important features of the plasma are regions of reversed-flow near the divertor plate which would tend to transport impurities upstream toward the $x$-point. Two such regions exist, one along the separatrix and one further out into the scrape-off layer, as shown in Fig. 1.

\section{B. Spatial Distribution of Impurity Radiation}

We illustrate the behavior of various impurities at low concentrations which do not appreciably disturb the hydrogenic background plasma.

For the recycling impurities, helium and neon, with a fixed core concentration of $.03 \%$, the radiation heat load on the outer wall is shown in Fig. 2. For helium, the total radiation is only $0.14 \mathrm{~kW}$, with $25 \%$-coming from the divertor leg and the remainder from a-broad halo in the outer scrape-off layer near the midplane. For neon, the total radiation is much larger, about $230 \mathrm{~kW}$ / batiansmaller fraction comes from the divertorleg because few neon impurity ions reach the divertor plate.

For the non-recycling impurities, beryllium and carbon, with a sputtering yield of $.01 \%$, essentially all of the radiation originates from within a few centimeters of the divertor plate. The total impurity radiation is much larger for beryllium $\left(660^{-k} \mathrm{~kW}\right)$ than for carbon $(240 \mathrm{~kW})$. Some small fraction of the impurities generated at the divertor plate escape to the core region. This fraction is about $0.4 \%$ for beryllium and $1.5 \%$ for carbon. The tendency for the carbon to escape more readily is seen in the broader radiation source near the divertor plate shown in Fig. 3. This may be explained by the somewhat higher ionization energy of the neutral carbon atom which allows it to penetrate upstream to regions of higher electron temperature, and the thermal and friction forces (reversed flow) being proportional to $Z^{2}$.

\section{Solutions with Strong Sputtering}

The carbon plate sputtering yield was arbitrarily increased from zero to a maximum yield of $0.01(1 \%)$, with all other model parameters fixed. In Fig. 4 we show the variation 
of the total impurity radiation, the carbon contribution to $Z_{\text {eff }}$ at the midplane and the fraction of the sputtered carbon that escapes from the divertor. For very weak sputtering there is little carbon in the divertor and the buildup of carbon at the midplane is limited by our fixed-density boundary condition at the core. For strong sputtering, most of the radiation comes from a region just in front of the divertor plate where the total carbon density is a maximum and the electron temperature is low enough for efficient radiation by the lower charge states. The total impurity radiation for a $1 \%$ sputtering yield is about $30 \%$ of the power into the scrape-off layer, but the peak heat load on the divertor plate is not significantly reduced because the radiation is concentrated near the plate.

A similar scan of the sputtering yield for beryllium yields the results shown in Fig. 5 . For a given sputtering yield, the total impurity radiation from beryllium is more than 3 times larger than from carbon, and the fraction of the beryllium escaping from the divertor is less than half of the fraction for carbon. Note that the maximum sputtering yield for our beryllium simulations is considerably smaller than for our carbon simulations; with stronger sputtering, there is an increased tendency for the plasma to detach from the divertor plate and it is more difficult to obtain converged steady-state solutions.

$\therefore$ The above simulations all used a fixed-core-density constraint on the impurities, mainly because convergence to steady state solutions is more robust with this boundary condition. We relaxed this condition for a series of carbon sputtering simulations by allowing the upstream carbon density to adjust until the net impurity flux across the core boundary is zero. This corresponds to no net injection or removal of carbon from the system. Carbon flows upstream along flux surfaces to the midplane where it diffuses radially into the core; the carbon in the core distributes itself more or less uniformly along flux surfaces, and the carbon diffuses radially outward from the core near the $x$-point where the carbon density is relatively low. For a carbon plate sputtering yield $\mathrm{Y}_{\text {phys }}=.0015$, we find the carbon density in the core rises to $2.7 \times 10^{17} \mathrm{~m}^{-3}(0.53 \%$ concentration $)$ and the maximum $Z_{\text {eff }}=$ 1.2 along the separatrix. This core impurity concentration and the total impurity radiation vary with the sputtering yield as shown in Fig. 6. The equilibrium core concentration may also depend on the strength of the radial particle diffusion, which for these simulations was $D_{\perp}=0.33 \mathrm{~m}^{2} / \mathrm{s}$ for both hydrogen and carbon. For this series of runs, the core hydrogen plasma density was higher $\left(5 \times 10^{19} \mathrm{~m}^{-3}\right.$ versus $\left.3 \times 10^{19} \mathrm{~m}^{-3}\right)$ than in the above simulations 
and the divertor plate was moderately inclined rather than strictly orthogonal.

\section{Inter-species Charge Exchange}

Our standard model assumes equal rate parameters for the two interspecies charge exchange interactions between deuterium $(D)$ and carbon $(C)$ :

$$
\begin{aligned}
& D+C^{+} \rightarrow D^{+}+C \\
& C+D^{+} \rightarrow C^{+}+D
\end{aligned}
$$

Rates for reaction (1) are available in the tables we use, ${ }^{15}$ but there are no values for reaction (2). Since the rate parameter for reaction (2) is uncertain, we tested the sensitivity of our model results by arbitrarily reducing this rate for a case with weak carbon sputtering, $Y_{\text {phys }}=.0001$. This has the effect of increasing the mean free path of the impurity gas, allowing it to penetrate further upstream from the divertor plate. We see this in the results shown in Table I.

Table I: Effect of Reduced Charge Exchange for Carbon Neutrals

\begin{tabular}{|l|l|l|l|}
\hline CX Reduction & $Z_{\text {eff }}$ from C & Escape Fraction & Carbon Radiation (MW) \\
\hline 1.00 & .0046 & .015 & 0.23 \\
\hline 0.50 & .0061 & .035 & 0.65 \\
\hline 0.25 & .0086 & .069 & 1.40 \\
\hline 0.00 & .0145 & .158 & 4.13 \\
\hline
\end{tabular}

A $50 \%$ reduction in the rate parameter causes a $50 \%$ increase in the carbon concentration near the midplane and a factor of two increase in the fraction of carbon ions which escape from the divertor region and enter the core plasma rather than return to the divertor plate. We also find that the total carbon radiation increases by a factor of three in response to this rate reduction. Setting the rate parameter to zero has even stronger consequences, so it is important to get more accurate information about this inter-species charge exchange rate.

We have repeated calculations on the effect of reduce carbon neutral charge exchange for the $30^{\circ}$ inclined plate case. The results are quantitatively the same for the factor of 2 reduction in the rate seen in Table 1 . 
For recycling impurities, such as neon, the effect of inter-species charge exchange reaction (2) is much weaker than for carbon. This reaction mainly affects the mean-free-path and density of the neutral impurity. For neon, most of the impurity ions simply circulate within the scrape-off layer without ever reaching the divertor plate, so there is very little neon gas in the divertor. For carbon, on the other hand, essentially all impurity ions in the scrape-off layer originate as neutral carbon sputtered from the divertor plate by incident hydrogen ions. Our model results show that there is no significant change in the midplane $Z_{\text {eff }}$ or in the total neon radiation when we vary the charge exchange rate (2) reduction factor from 1 to 0 .

\section{Behavior as Detached Solution Is Approached and Comparison of Impurity Models}

As reported in Ref. 1 , we found that we could find steady-state solutions which radiated roughly $25 \mathrm{MW}$ in the outer divertor leg ( $\sim 40 \mathrm{MW}$ for both legs) for $100 \mathrm{MW}$ crossing

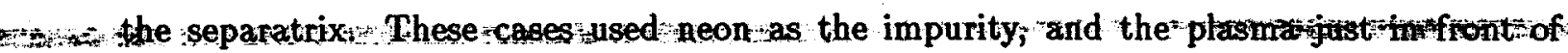
the divertor plate was typically a few $\mathrm{eV}$ in temperature, but not completely detached. For higher impurity levels; no steady-state solution could be found-At the same-power level where steady-state solutions were lost, a MARFE just under the $x$-point was growing in strength, $=$ To understand this behavior in more detail, and the calibrate the multicharge-state (MCS) model with the more rapid-running fixed-fraction (FF) model used later for some of the time-dependent calculations, we have done a series of systematic studies comparing the two impurity models and the resulting impurity radiation obtained.

The case considered has a core hydrogen density of $5 \times 10^{19} \mathrm{~m}^{-3}$, and $100 \mathrm{MW}$ into the outer-half of the SOL, equally divided between ions and electrons. The anomalous diffusion coefficients again correspond to the so-called weak diffusion case ${ }^{1}$ with $D_{\perp}=0.33 \mathrm{~m}^{2} / \mathrm{s}$ and $\chi_{e, i}=0.5 \mathrm{~m}^{2} / \mathrm{s}$. Because we wish to examine the $\mathrm{x}$-point region in some detail for this study, we improved the resolution of the mesh near the $x$-point; however, the basic conclusions are essentially the same as for the less refined mesh used in Ref. 1 . In this section, we consider an orthogonal divertor plate with no pumping to illustrate the main points. However, in Sec. IVB, we return to an nonorthogonal mesh with a vertical divertor 
plate.

To represent the spatial distribution and effectiveness of the radiating impurities, we integrate over the radial dimension for the power radiated by the impurity and plot the result versus poloidal location as measured by distance along the separatrix. Such a plot is shown in Fig. 7a for the FF model with neon using coronal equilibrium for the three fraction values of $n_{z} / n_{e}=0.025 \%, 0.05 \%$, and $0.1 \%$. In Fig. $7 \mathrm{~b}$, we show results for the MCS model using three different values of the core boundary density for charge-state $+8: n_{z}^{+8} / n_{e}=$ $0.2 \%, 0.4 \%$, and $0.5 \%$. The radiated power for these six cases in shown in Table II; note that the last near tripling in power occurs between the last two table entries for only a $25 \%$ increase in core impurity density. At a given impurity fraction at the core boundary, the FF model overestimates the power radiated because the impurity distribution is very nonuniform spatially. For both models, the impurity levels cannot be increased by another $25 \%$ above the highest value used here and still find steady-state solutions. We shall return to this limit for our discussion of oscillations in Sec. IVA.

Table II: Comparison of Models for Neon in Outer-Half of ITER

\begin{tabular}{|c|c|c|}
\hline Model & Impurity fraction at core edge & Power radiated (MW) \\
\hline \multirow{3}{*}{ Fixed-fraction } & $0.025 \%$ & 7.8 \\
\cline { 2 - 3 } & $0.05 \%$ & 19.4 \\
\cline { 2 - 3 } & $0.1 \%$ & 39.3 \\
\hline \multirow{2}{*}{ Multi-charge state } & $0.2 \%$ & 3.7 \\
\cline { 2 - 4 } & $0.4 \%$ & 9.0 \\
\cline { 2 - 4 } & $0.5 \%$ & 26.0 \\
\hline
\end{tabular}

There are several points to be made from the plots of the power versus poloidal distance: (1), the FF model shows a roughly linear dependence of power on the impurity fraction. (2), In contrast, the MCS model shows a very nonlinear dependence of radiated power with impurity level. This is caused by the localization of the impurity near the midplane for low levels owing to hydrogen back flow and thermal forces, and the overcoming of these effects at higher impurity levels via changes in the plasma profiles. (3), both models show significant radiation just below the $\mathrm{x}$-point near $x \approx 12.3 \mathrm{~m}$, with the FF model giving a larger effect.

Thus, at low impurity levels, the two models differ substantially owing to 2-D transport 
of the impurities, but the qualitative features become more similar at the higher levels when we used the total power radiated as the common measure. As noted in Ref. 1, the MCS model also shows better spatial mixing for tilted divertor plates where a large-scale circulating flow develops in the SOL. Different parameters will also affect this comparison. In particular, using UEDGE with a core density of $4 \times 10^{19} \mathrm{~m}^{-3}$ and anomalous diffusion coefficients of $1 \mathrm{~m}^{2} / \mathrm{s}$, Wising, et al. ${ }^{17}$ found steady-state solutions for $0.8 \%$ neon in the FF model resulted in $78 \mathrm{MW}$ of power radiated in the outer-half of ITER, and we have verified this result. While we have not done the detailed MCS model comparison, our strong diffusion case reported in Table II of Ref. 1 with a core hydrogen density of $5 \times 10^{19} \mathrm{~m}^{-3}$ and anomalous diffusion of $D=1 \mathrm{~m}^{2} / \mathrm{s}$ and $\chi_{e, i}=0.67 \mathrm{~m}^{2} / \mathrm{s}$ gave a MCS radiated power of $18 \mathrm{MW}$ for the same core impurity level of $0.8 \%$. This difference is in semi-quantitative agreement with the comparison in Table II in this report. Overall, the results show that basing quantitative design on the FF model can be subject to large error. However, the more efficient FF model can help us to better understand the basic dynamics and classify different types of plasma behavior for the ITER geometry as discussed in the next section.

\section{Time-Dependent-Solutions and Detachment}

When the impurity level is raised above the maximum levels given in the previous section for either impurity model, asteady-state solutionsware y difficult to obtain, requiring a delicate balance of particle input and pumping. More generally, two types of time-dependent solutions result depending on the core density boundary condition. The first keeps the total particle content fixed by having no particle flux from the core and no pumping, and then oscillatory solutions appear, at least near the attached/detached transition. The second type of solution results when we use a fixed density at the core boundary, and the plasma slowly evolves deeper into detachment with the ionization front moving upstream; here sufficient pumping can move the front back toward the divertor plate. We now illustrate these two types of solutions for our ITER cases. 


\section{A. Oscillatory Solutions}

If we increase the concentration of neon from either the FF model or the MCS model more than $25 \%$ from the largest values shown in Table II, we cannot find a steady-state solution without pumping. If we use our typical core boundary condition of fixed density, there is a continuous flow of particles across the core boundary leading to deeper detachment; this behavior is shown in the next subsection. Here we consider the case where the particle flux at the core boundary is forced to zero such that the total number of hydrogen particles (ions+neutrals) remains constant. Note that for the steady-state solutions with no pumping described previously, these two boundary conditions lead to the same solution, i.e., even if we specify the density, zero flux across the core boundary is obtained at steady state because there is no pumping.

To illustrate the oscillatory solution, we begin from the steady-state solution shown in Table II and Fig. 7 having $0.1 \%$ fixed-fraction of neon. The neon fraction is then doubled to $\mathbf{0 . 2 \%}$, and a zero density flux boundary condition is imposed at the core boundary. After some initial transients, the solution exhibits regular oscillations with a period of $\sim 18 \mathrm{~ms}$ The effect on the solution is shown by plots of the hydrogenic ion density and electron temperature at different points along the SOL as a function of time in Fig. 8 for a time of $36 \mathrm{~ms}$ which shows two periods of the oscillation. The time-dependent plots are shown at two radial locations corresponding to magnetic flux surfaces $0.2 \mathrm{~cm}$ and $1.4 \mathrm{~cm}$ outside of the separatrix as measured at the divertor plate. The oscillations are largest near the separatrix and in the private flux region. The $1.4 \mathrm{~cm}$ surface is at about the peak of the heat flux on the plate. In addition, we show the poloidal profile of impurity radiation at four time-slices over the oscillation in Fig. 9. This shows the substantial involvement of the radiation from the $\mathrm{x}$-point region in the oscillation; whether this radiation is helping drive the oscillation or just responding has not yet been determined.

These oscillations are likely similar to those discussed earlier by Krasheninnikov, et al., ${ }^{16}$ except that here we also have the involvement of the $x$-point radiation. Near the divertor plate there is a cyclic conversion of neutrals into ions and then back to neutrals. This is strongest near the private-flux/separatrix boundary where power to ionize the neutrals is limited by the radial diffusion of energy into the private flux region. Note that the ion 
density oscillations are very large at the plate, even for the $1.4 \mathrm{~cm}$ flux surface where the other oscillations are relatively weak. This is also true of the neutral density, so to properly model these oscillations, one must follow the neutrals time-dependently.

\section{B. Slowly Evolving Detached Solutions}

The second type of solution we find at increased impurity levels uses the fixed-hydrogenicdensity core boundary condition. In this case, the solution appears to go through an oscillatory phase as it first passes into complete detachment, but then evolves (possibly with small oscillations) to a more deeply detached state where the ionization front moves farther upstream from the divertor plate. This behavior has been observed for both FF and MCS impurity models, and for an orthogonal divertor plate as well as one inclined $90^{\circ}$ in the $(R, Z)$ plane. Here, we shall focus on the results from the most complete model, i.e, the MCS impurity model with the $90^{\circ}$ plate.

An additional refinement to the model is that the neutral gas is allowed to escape along the private flux boundary with an albedo of 0.5 (about $1 / 2$ sonic flow) This gas is then reinjected along the private flux boundary with a fixed profile, usually taken to be a cosine factor having a maximum at the plate, and falling to zero at $25 \mathrm{~cm}$ below the $x$-point to simulate the baffle proposed for use under the $x$-point. This model simulates the mixing of the neutral gas in the plenum in the private flux region. An example of how this model redistributes the gas for the time slice at $0.05 \mathrm{~s}$ is given in Fig. 10. Here the largest flux leaves the plasma region near the divertor plate, and is rather uniformly distributed over

-... the middle $2 / 3$ of the private flux boundary. To simulate pumping with this model, one simply reinjects a fraction of the escaping particle flux.

The two time slices shown in Fig. 11 evolved from an initial state having a core boundary condition for neon with the density $n_{z}^{+8}=2 \times 10^{17} \mathrm{~m}^{-3}$ and a hydrogenic core density of $5 \times 10^{19} \mathrm{~m}^{-3}$. At $\mathrm{t}=0 \mathrm{~s}$, the value of $n_{z}^{+8}$ is instantaneously raised to $4 \times 10^{17} \mathrm{~m}^{-3}$, and the solution evolved time-dependently. After some initial transients, the ionization front (as measured roughly by the $T_{e}=5 \mathrm{eV}$ contour), moves upstream from the divertor plate in a monotonic fashion as shown by the time slice at $t=0.05 \mathrm{~s}$ in Fig. 11(a). At a much later time (0.4 s), the ionization front is half way up the divertor leg as shown in Fig. 11(b). 
These evolving solutions are characterized by strong ionization and recombination radiation losses combined with neon radiation loss, and have negligible power reaching the divertor plate. These solutions appear to be the same type as found by Kukushkin, et $a l^{18,19}$ at similar impurity densities in their B2/EIRENE ITER modeling. The following table displays some of the important properties of the solutions:

Table III: Properties of Detached Plasmas at Two Times

\begin{tabular}{|l|l|l|l|c|c|}
\hline Time (s) & $P_{i z}$ & $P_{r c}$ & $P_{i m p}$ & $N_{e}$ & $N_{n}$ \\
\hline \hline 0.05 & 21 & 32 & 35 & $1.2 \times 10^{22}$ & $9.5 \times 10^{21}$ \\
\hline 0.40 & 29 & 37 & 29 & $1.5 \times 10^{22}$ & $5.5 \times 10^{22}$ \\
\hline
\end{tabular}

Here $P_{i z}, P_{r c}$, and $P_{i m p}$ are the powers in MW radiated by hydrogenic ionization, hydrogenic recombination, and neon radiation, respectively. The quantities $N_{e}$ and $N_{n}$ are the total number of electrons and hydrogenic neutrals in the outer-half computational domain, respectively; the volume of the domain is $144 \mathrm{~m}^{3}$.

As one can see from Table III, the number of neutral particles in the system is growing with time. These particles come from an ion flux across the core boundary which remains in the range of $1 \times 10^{23}$ particles/s over the course of the run; these ions eventually recombine below the ionization front after many ionization/recombination events in the front. The build-up of the neutral gas below the front pushes the ionization front slowing toward the $\mathrm{x}$-point. This is essentially the gas-target divertor solution discussed earlier. ${ }^{20,21}$ The timescale of the evolution corresponds to the time for the core ion frux to fill the lower divertor region with gas at a density of $\sim 4 \times 10^{21} \mathrm{~m}^{-3}$ :

$$
t \sim 4 \times 10^{21} \mathrm{~m}^{-3} \times 10 \mathrm{~m}^{3} / 1 \times 10^{23} \mathrm{~s}^{-1} \sim 0.4 \mathrm{~s},
$$

where the factor $10 \mathrm{~m}^{3}$ is the volume of the divertor leg below the ionization front in Fig. 11(b).

We have found these evolving, detached solutions for orthogonal plates and with or without gas recirculation in the private flux region. Steady-states have been obtained by pumping gas at the rate corresponding to the ion flux across the core. However, this ion flux is non physical in that it exceeds the available core particle source by more than an order of magnitude. We have begun some simulations with the core ion flux set to zero, and will report on them in our next status report. As discussed in Sec. IVA, such a boundary condition results in strong oscillations for barely detached plasmas, but the details may 
differ for these strongly detached cases. It may also be possible to maintain the detached state by puffing gas on the wall side at the midplane, and then pumping in the divertor region; we shall also consider these cases.

These detached states are attractive for helium pumping, and we have assessed this by including helium in a trace limit for the detached case shown in Fig. 11(b). Two results are noted here: First, the power radiated by the helium is $4.5 \times\left(100 n_{z}^{+2} / n_{e}\right) \mathrm{MW}$ where the density ratio is at the core boundary (e.g., $2 \%$ helium radiates $9 \mathrm{MW})$. Second, these strongly detached plasmas have high neutral densities with the helium gas density on the private flux boundary relative to the helium ion density at the core boundary being $n_{g, H e} / n_{z}^{+2} \approx 12$; this compares to the ratio of the deuterium species of $n_{g, D} / n_{i} \approx 80$. For this case, the ratio for neon is about the same as for helium, i.e., $n_{g, N e} / n_{z}^{+8} \approx 12$, where $n_{z}^{+8}$ is the highest density neon charge-state on the core boundary.

\section{Summary and Conclusions}

At low impurity concentrations, we find steady-state solutions for the edge plasma. Radiative losses from recycling impurities, $\mathrm{He}$ and $\mathrm{Ne}$, are spread over the entire length of the divertor and SOL, whereas the radiation from non-recycling sputtered impurities, Be and $\mathrm{C}$, is concentrated within a few centimeters of the divertor plates for attached plasmas. For non-recycling impurities, we find the solutions are sensitive to charge exchange between the neutral impurity atom and hydrogenic ions near the divertor plate. More accurate information about this charge exchange reaction is needed.

For higher concentrations of recycling impurities, such as neon, most of the power into the SOL is radiatively lost and steady-state plasma solutions may not exist. We find two types of evolving plasmas, depending on the flux of hydrogenic ions across the core boundary: (1) for fixed core density, the detached ionization front slowly moves upstream from the divertor plate on a time scale of several hundred milliseconds; (2) for zero particle flux across the core boundary, we find solutions which oscillate between weakly attached and strongly detached on a time scale of about 20 milliseconds. The oscillations can be accompanied by a MARFE under the x-point. The slowing evolving solutions can be stabilized by pumping in the divertor region. However, we must first understand the behavior of the 
detached solutions when the core ion flux is constrained to proper values.

The focus of our continuing work will be:

1. Include helium and other intrinsic impurities in the non-trace limit together with the injected impurity such as neon.

2. Analyze the partially detached plasma and associated oscillations in more detail.

3. Simulate detached plasmas with constrained core ion flux, and devise pumping and puffing scenarios to maintain this state.

4. Compare Monte Carlo and fluid neutral models for detailed ITER configuration.

Acknowledgments: We thank Drs. A. Kukushkin, D. Post, and F. Wising for helpful discussions. 


\section{References}

${ }^{1}$ M.E. Rensink and T.D. Rognlien, "Modeling Impurities and Tilted Plates in the ITER Divertor: Part I", LLNL Rept. UCRL-ID-124702, July 29, 1996.

${ }^{2}$ S.I. Braginskii, Transport processes in a plasma Reviews of Plasma Physics, Vol. I, Ed. M.A. Leontovich (Consultants Bureau, New York, 1965), p. 205.

${ }^{3}$ T.D. Rognlien, J.L. Milovich, M.E. Rensink, and G.D. Porter, J. Nucl. Mat. 196-198, 347 (1992).

${ }^{4}$ D.A. Knoll, A.K. Prinja, and R.B. Campbell, J. Comp. Phys. 104, 418 (1992).

${ }^{5}$ T.D. Rognlien, P.N. Brown, R.B. Campbell, T.B. Kaiser, D.A. Knoll, P.R. McHugh, G.D. Porter, M.E. Rensink, and G.R. Smith, Contrib. Plasma Phys. 34, 362 (1994).

${ }^{6}$ G.R. Smith, P.N. Brown, R.B. Campbell, D.A. Knoll, P.R. McHugh, M.E. Rensink, and T.D. Rognlien, J. Nucl. Mat. 220-222, 1024 (1995).

${ }^{7}$ T.D. Rognlien, B.Ji=Braarns, and D.A. Knoll, Contrib. Plasma Phys. 36, 105 (1996).

${ }^{8}$ F. Wising, D.A. Knoll, S.I. Krasheninnikov, and T.D. Rognlien, Contr. Plasma Phys. 36, 309 (1996).

${ }^{9}$ D.A. Knoll, P.R. McHugh, S.I. Krasheninnikov, and D.J. Sigmar, Phys. Plasmas 3, 293 (1996).

${ }^{10}$ Yu.L. Igitkhanov, Contrib. Plasma Phys. 28, 477 (1988):

${ }^{11}$ M. Keilhacker, R. Simonini, A. Taroni, and M.L. Watkins, Nucl. Fusion 31, 535 (1991).

${ }^{12}$ S.P. Hirshman, private communication, 1995; base on work in S.P. Hirshman and D.J. Sigmar, Nucl. Fusion 21, 1079 (1981).

${ }^{13}$ D.P. Stotler, private communication, 1995; based on work initiated in D.P. Stotler, D.E. Post, and D. Reiter, Bull. Am. Phys. Soc. 38, 1919 (1993).

${ }^{14} \mathrm{~K}$. Behringer, "Description of the Impurity Transport Code STRAHL," JET Report $R(87) 08$ (1987). 
${ }^{15} \mathrm{~B} . J$. Braams, impurity rate tables for $\mathrm{B} 2.5$ code, available from CFS storage facility on the NERSC computer system, 1995.

${ }^{16}$ S.I. Krasheninnikov, A.S. Kukushkin, V.I. Pistunovich, and V.A. Pozharov, Nucl. Fusion 27,1805 (1987)

${ }^{17}$ F. Wising, S.I. Krasheninnikov, D.J. Sigmar, D.A. Knoll, T.D. Rognlien, B. LaBombard, B. Lipschultz, and G. McCracken, "Simulation of Plasma Flux Detachment in Alcator C-Mod and ITER,” J. Nucl. Mat., to be published, 1997.

${ }^{18}$ A.S. Kukushkin, H.D. Pacher, M. Baelmans, D. Coster, G. Janeschitz, D. Reiter, and R. Schneider, " 2-D Modelling of Radiative Divertor Regime for ITER,", J. Nucl. Mat., to be pub., 1997.

${ }^{19}$ A. Kukushkin, H. Pacher, et al., $16^{\text {th }}$ IAEA Fusion Energy Conference, Montreal, Canada, 7-11 Oct., 1996, paper FP-27.

${ }^{20}$ M. Petravic, Phys. Plasmas 1, 2207 (1994).

${ }^{21}$ D.A. Knoll, P.R. McHugh, S.I. Krasheninnikov, and D.J. Sigmar, Phys. Plasmas 3, 293 (1996). 


\section{Figures}

FIG. 1. Regions of reversed hydrogenic plasma flow in the outer divertor leg. The hydrogenic core density is $3 \times 10^{19} \mathrm{~m}^{-3}$ and the divertor plate is strictly orthogonal to flux surfaces.

FIG. 2. Impurity radiation heat load on the outer wall for low concentrations (.03\%) of helium and neon at the core. Note that the helium radiation is about 3 orders of magnitude smaller than that for neon.

FIG. 3. Impurity radiation source strength near the divertor plate for low sputtering yields $(.01 \%)$ of beryllium and carbon.

FIG. 4. For various carbon plate sputtering yields, we show the total impurity radiation, fraction of impurities escaping to the core, and impurity contribution to $Z_{\text {eff }}$ in the core.

FIG. 5. For various beryllium plate sputtering yields, we show the total impurity radiation, fraction of impurities escaping to the core, and impurity contribution to $Z_{\text {eff }}$ in the core.

FIG. 6. For various carbon plate sputtering yields with the zero-net-flux boundary condition at the core, we show the total impurity radiation, equilibrium impurity core concentration, and impurity contribution to $Z_{e f f}$ in the core: The hydrogenic core density is $5 \times 10^{19} \mathrm{~m}^{-3}$ and the divertor plate is slightly inclined relative to an orthogonal ' plate.

FIG. 7. Neon impurity power per unit length versus the poloidal distance along the separatrix for the cases shown in Table III; (a), for the fixed-fraction model, and (b), for the multi-charge-state model. Note that this is local emissivity, and not the net heat load on the wall as is shown in Fig. 2.

FIG. 8. One period of oscillations of the density and electron temperature at various poloidal locations on two different magnetic flux surfaces measured by distance from the separatrix on the divertor plate; a), $0.2 \mathrm{~cm}$, and b), $1.4 \mathrm{~cm}$. The poloidal positions are indicated by the letters $m$ (midplane), $x$ (x-point), $d 2$ (half-way between $x$-point and divertor plate), and $\mathrm{p}$ (divertor plate). 
FIG. 9. The neon radiation integrated radially at four times during the oscillation shown in Fig. 8 with $0.2 \%$ neon usin the FF model. Compare with Fig. $7 a$.

FIG. 10. The flux of deuterium on the private flux boundary versus poloidal location showing the escaping flux, the redistributed reinjected flux with a cosine profile, and the net flux, the latter being the sum of the former two. Corresponds to the time of $0.05 \mathrm{~s}$ for the evolving detached case in Fig. 11.

FIG. 11. Electron temperature profiles for the MCS model with a neon density at the core boundary of $n_{z}^{+8}=4 \times 10^{17} \mathrm{~m}^{-3}$. The hydrogenic density is fixed at the core boundary to $5 \times 10^{19} \mathrm{~m}^{-3}$. The slow evolution is caused by a small, but steady particle flux from the core. 


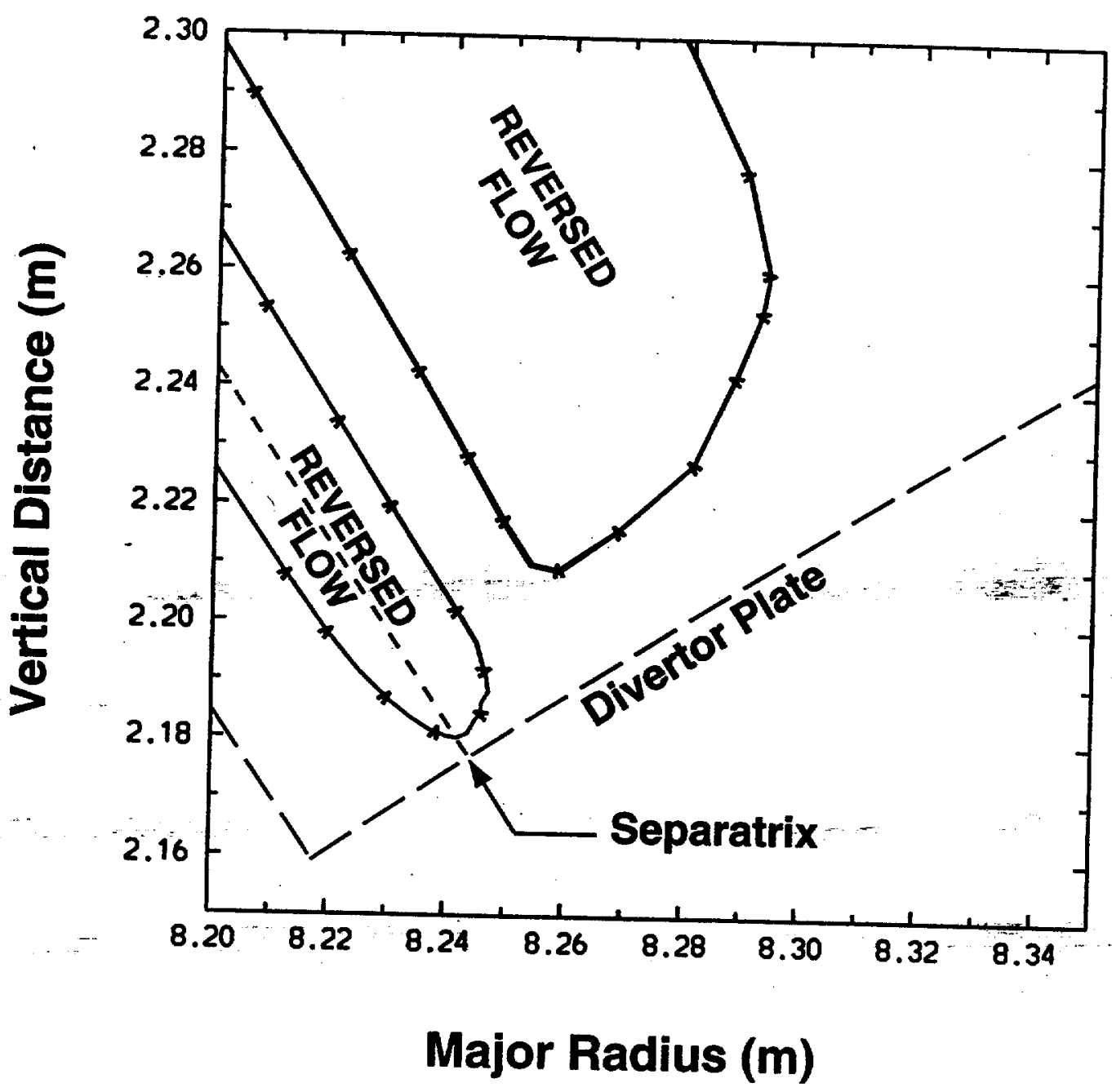

Figure 1 


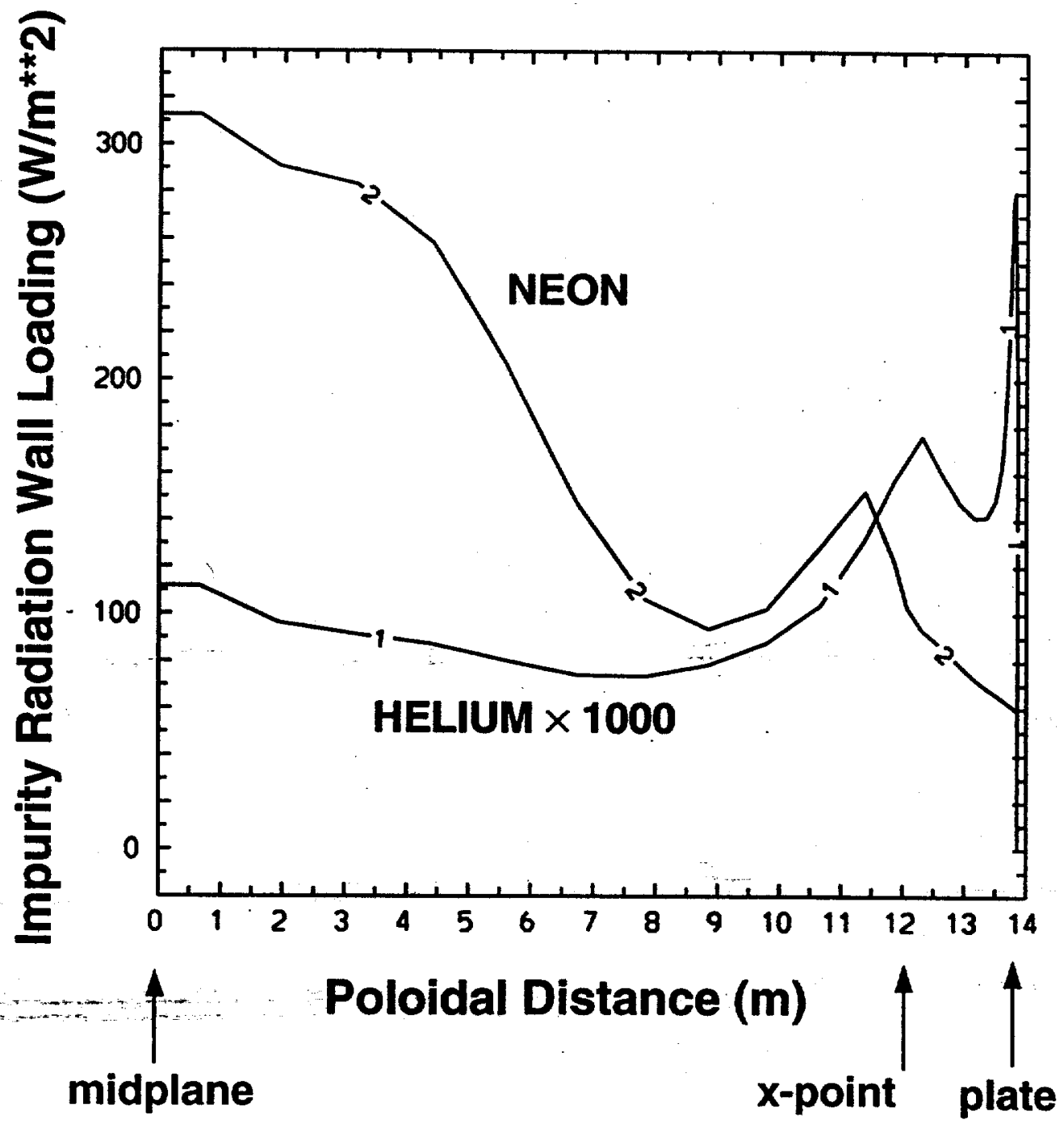

Figure 2 


\section{IMPURITY RADIATION SOURCE (MW/m*3)}

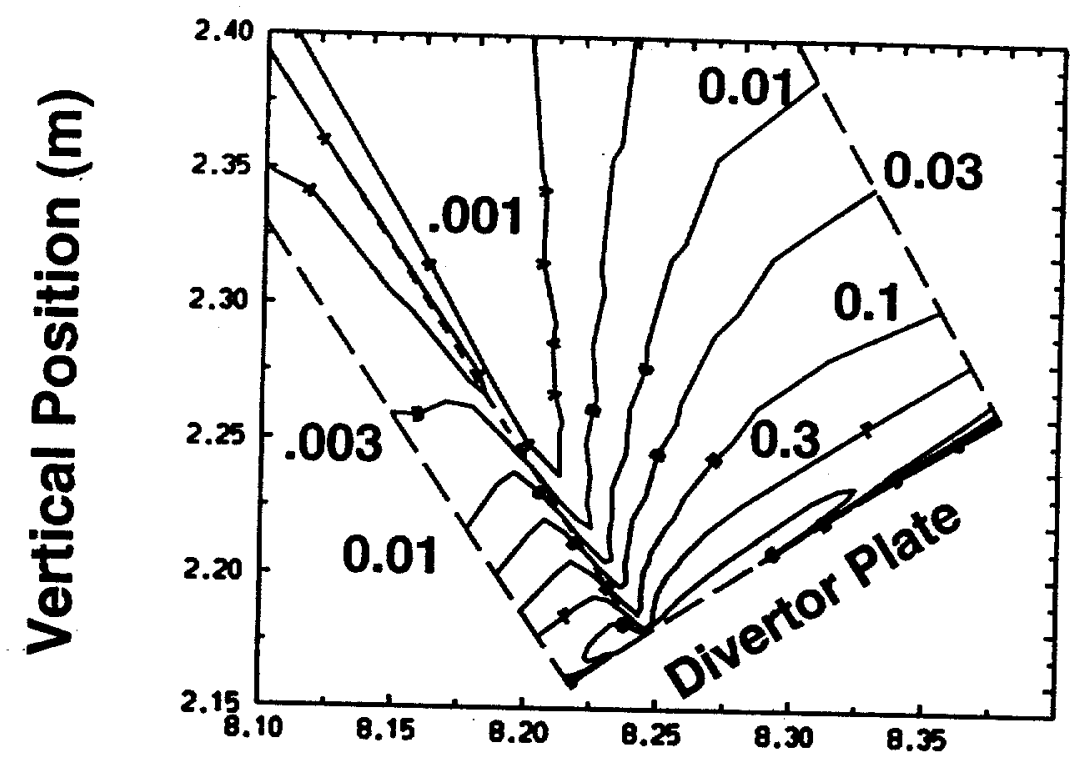

\section{CARBON}

\section{Major Radius (m)}
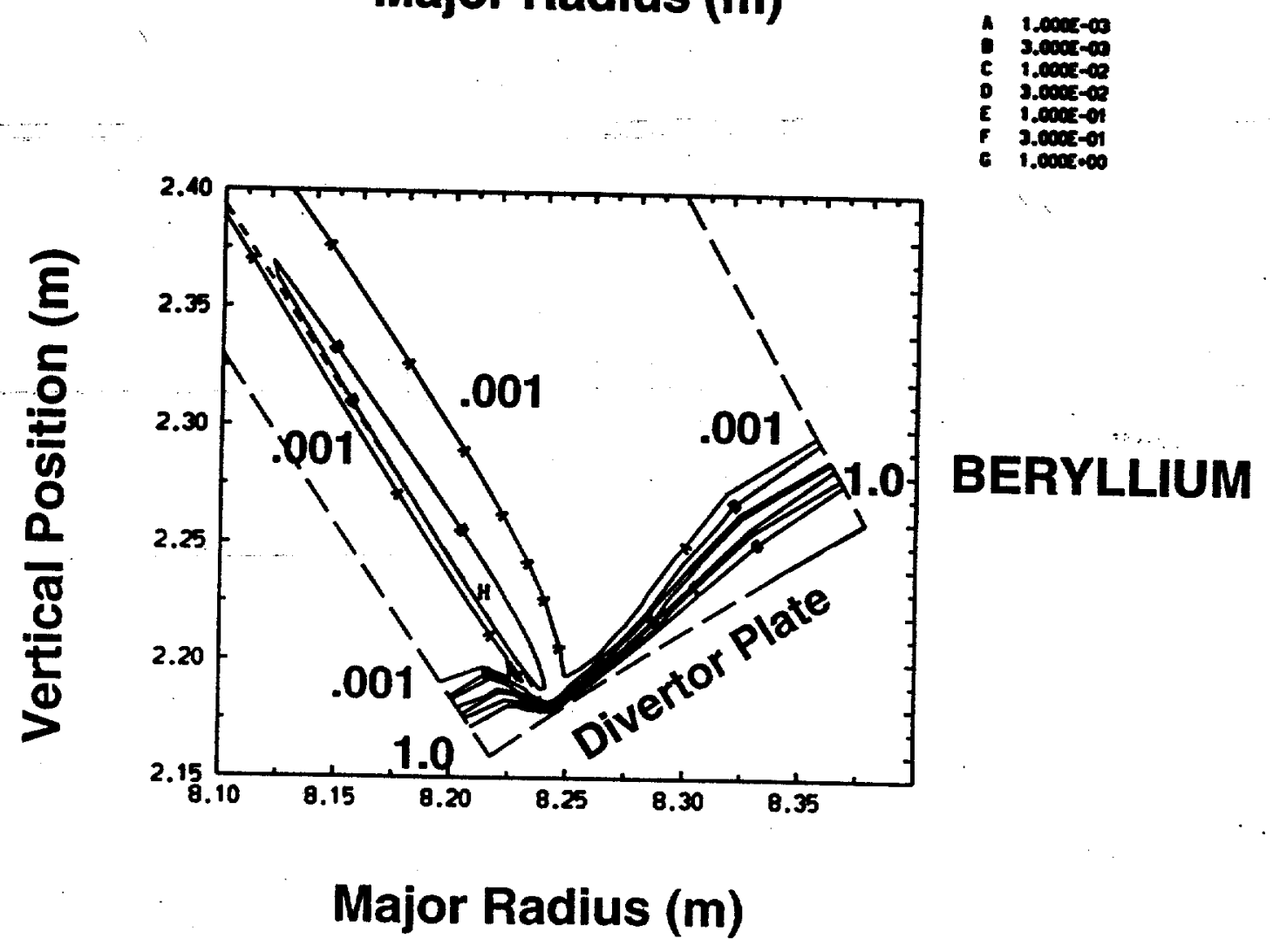

Figure 3 


\section{Orthogonal Carbon Plate}

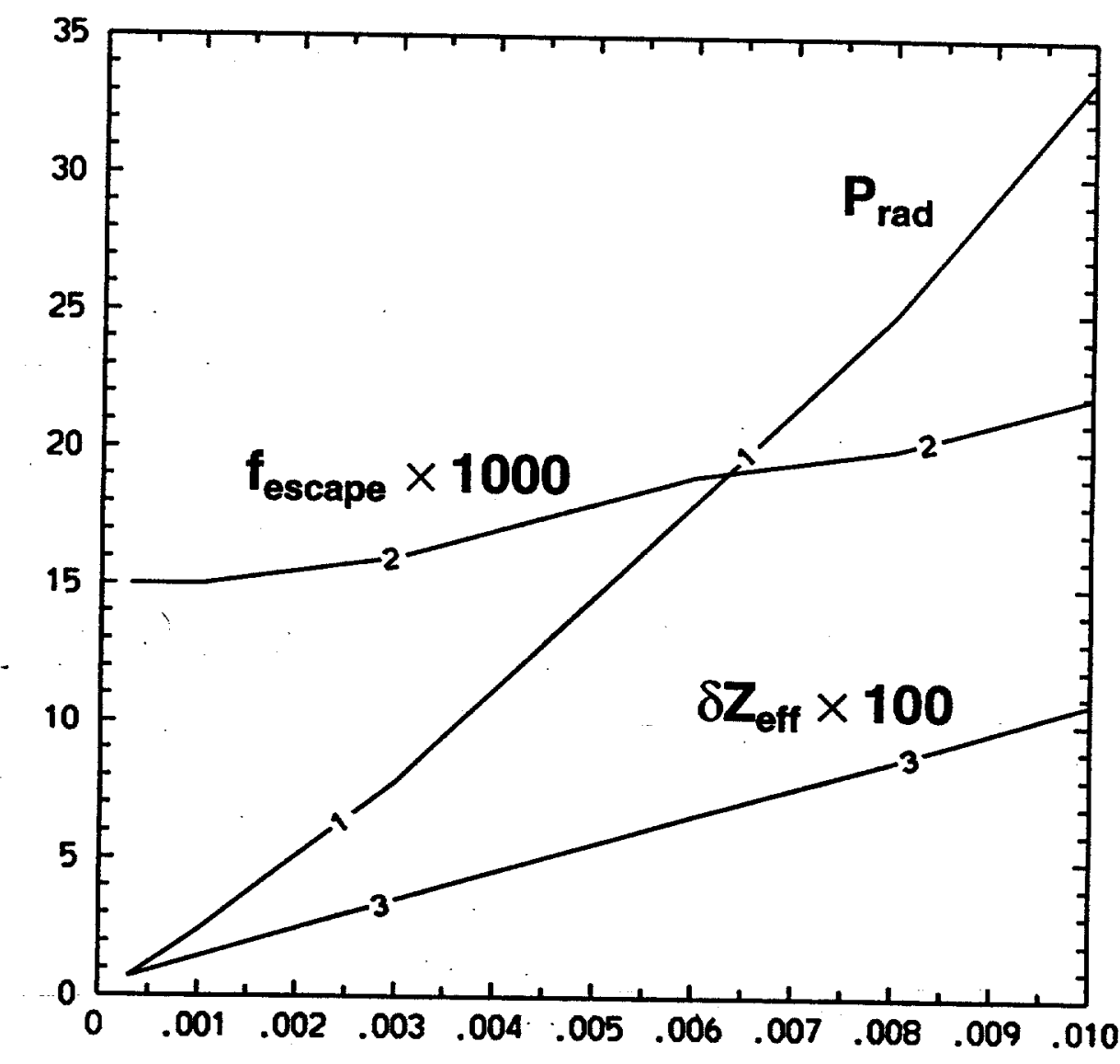

Sputtering Yield

Figure 4 
Orthogonal Beryllium Plate

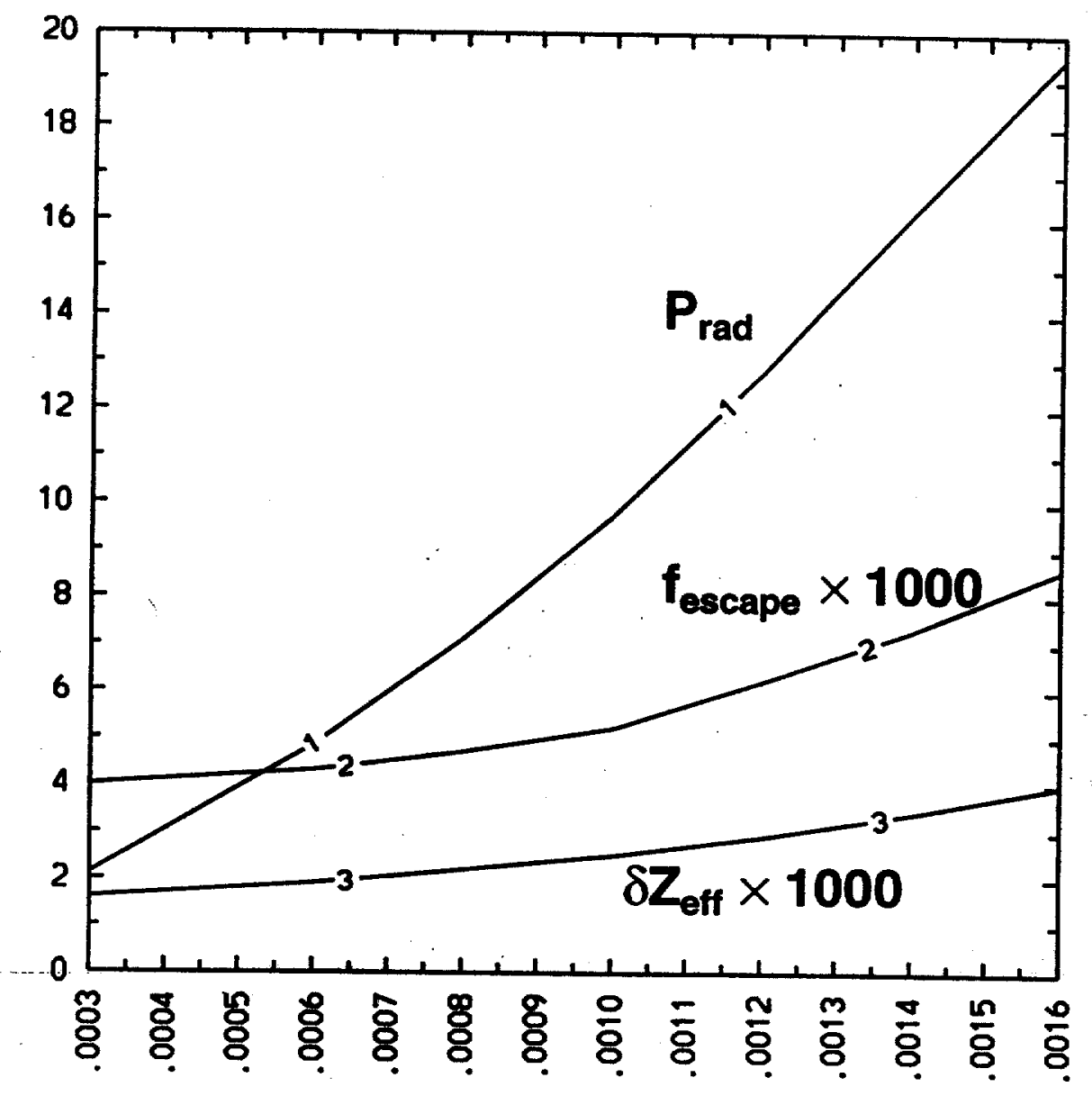

Sputtering Yield

Figure 5 


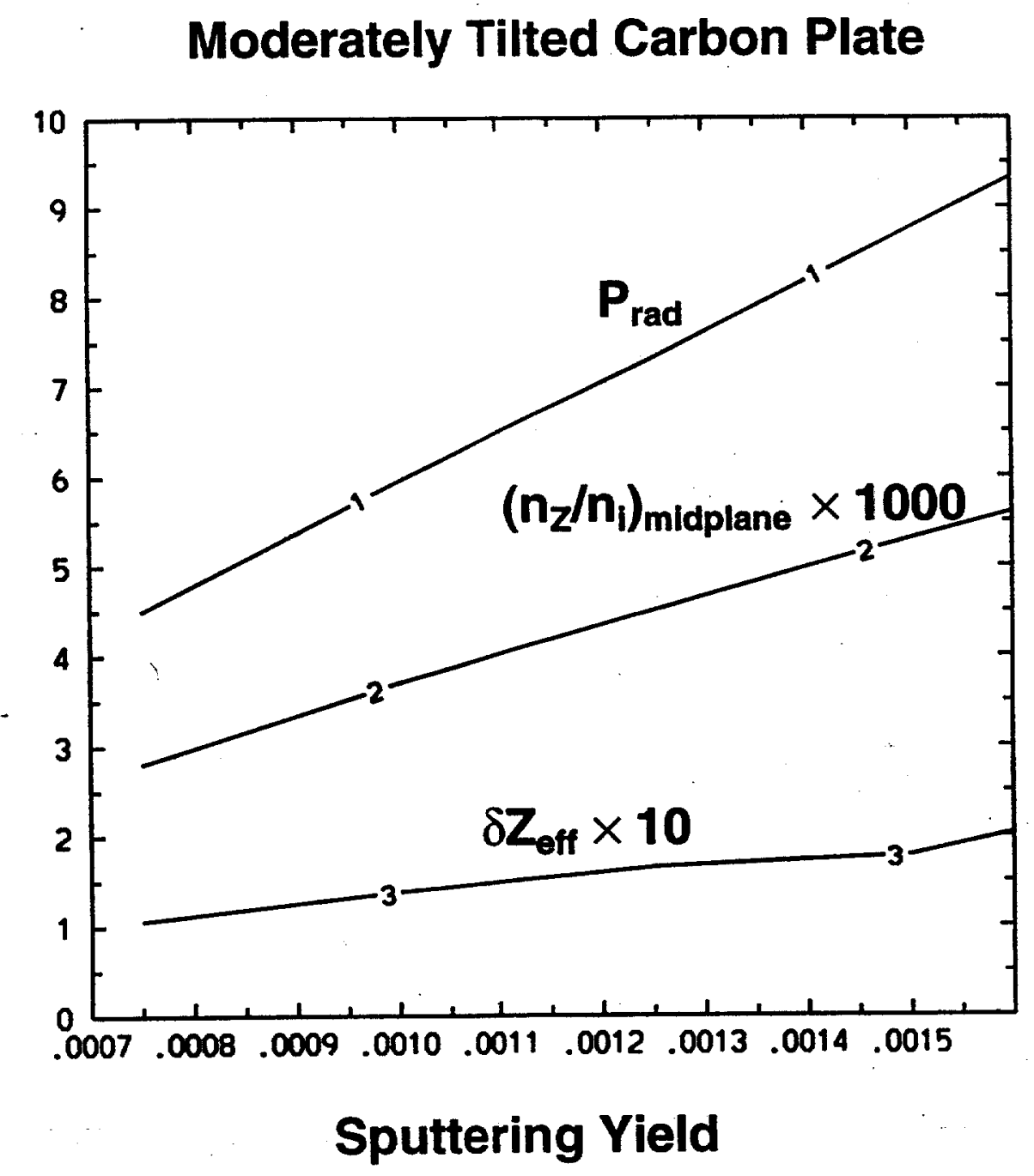

Figure 6 

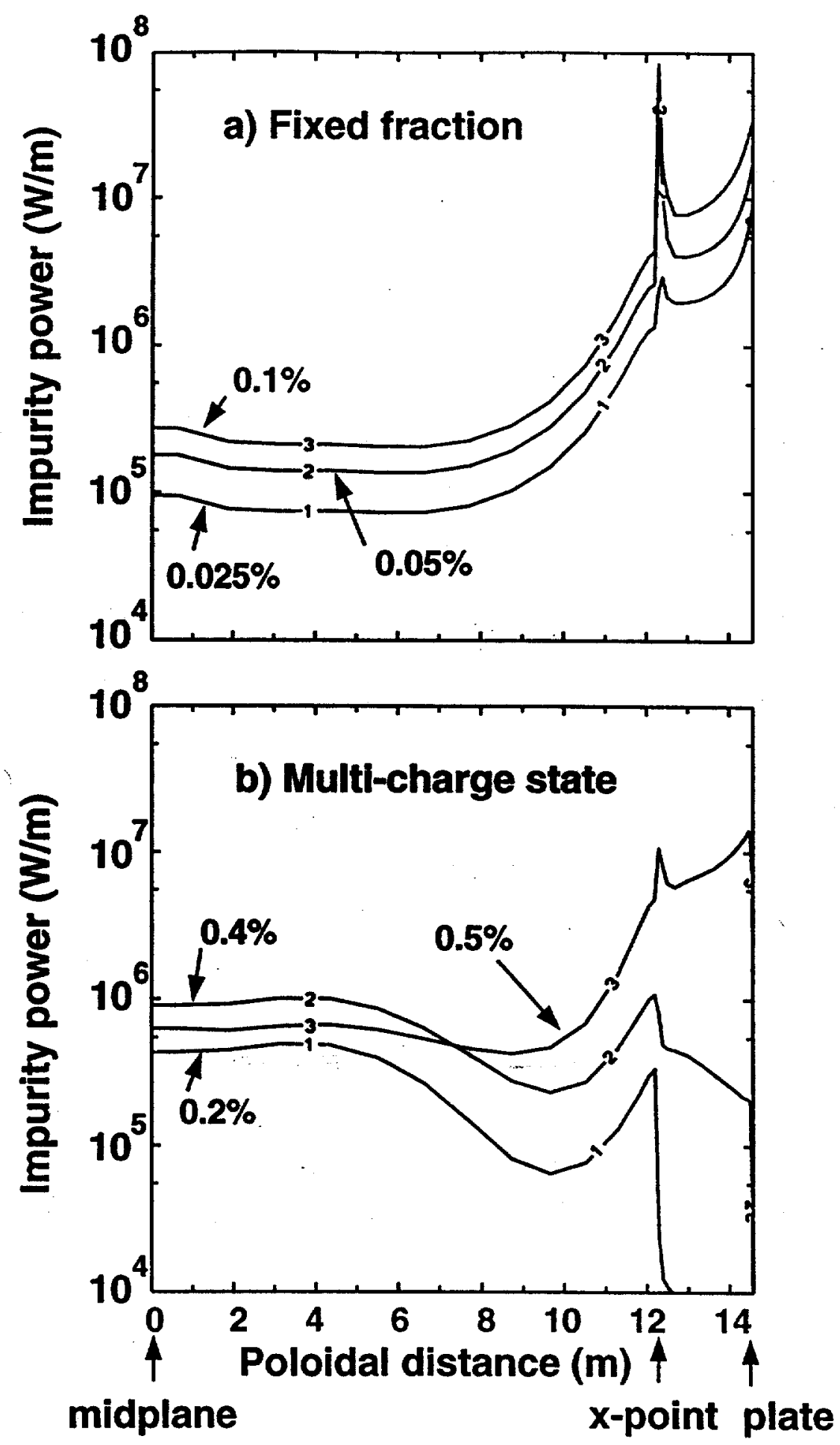

Figure 7 
a)
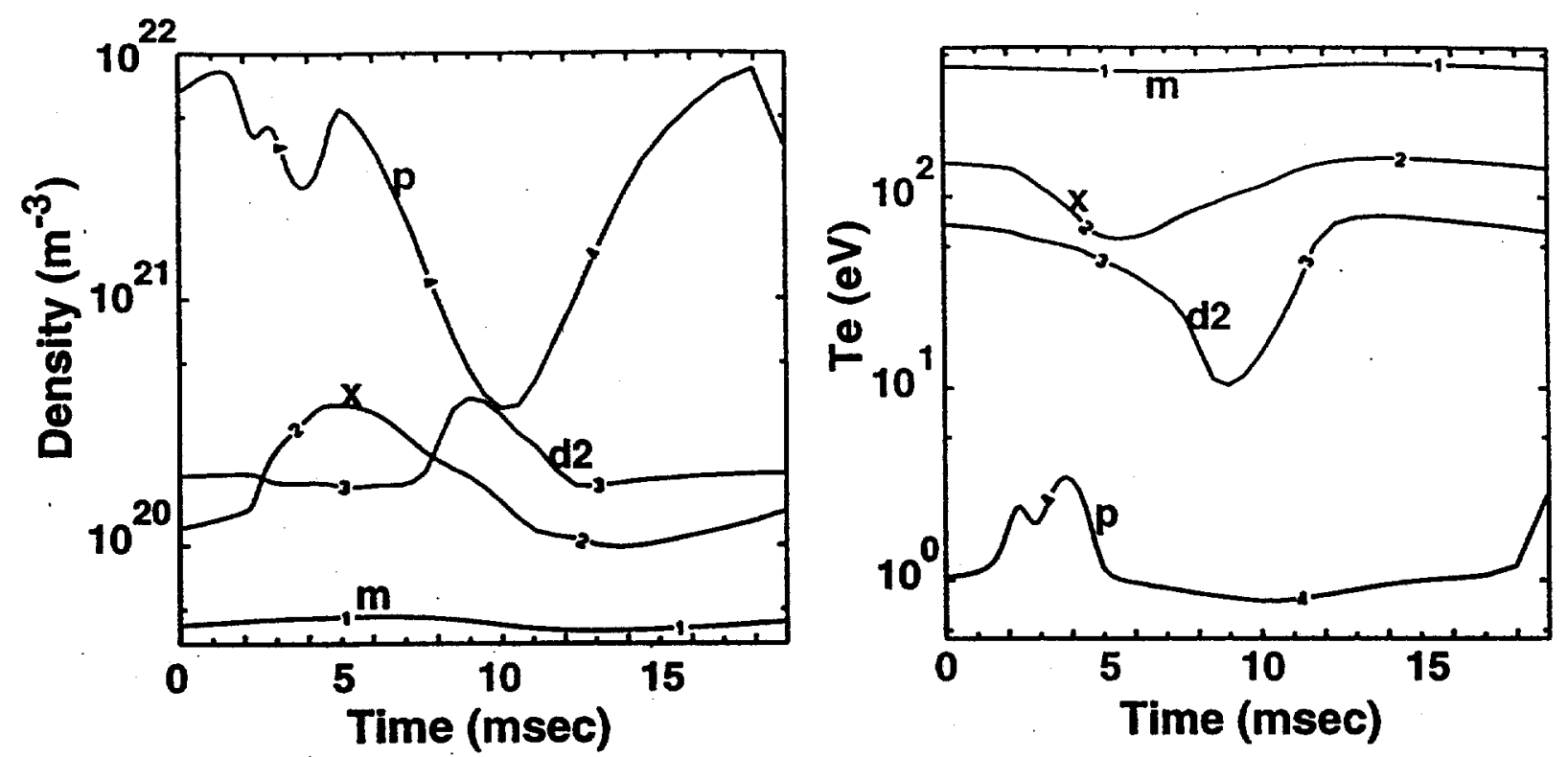

...
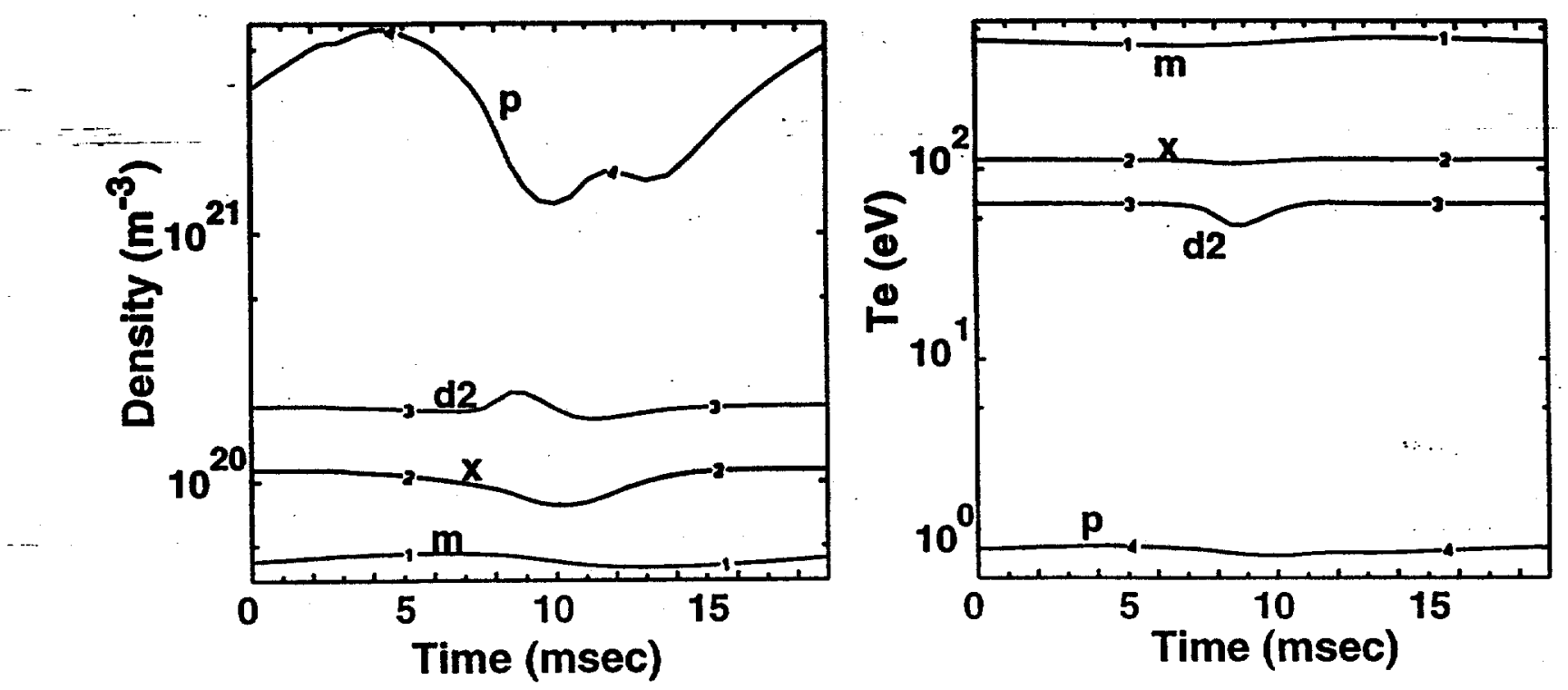

Figure 8 


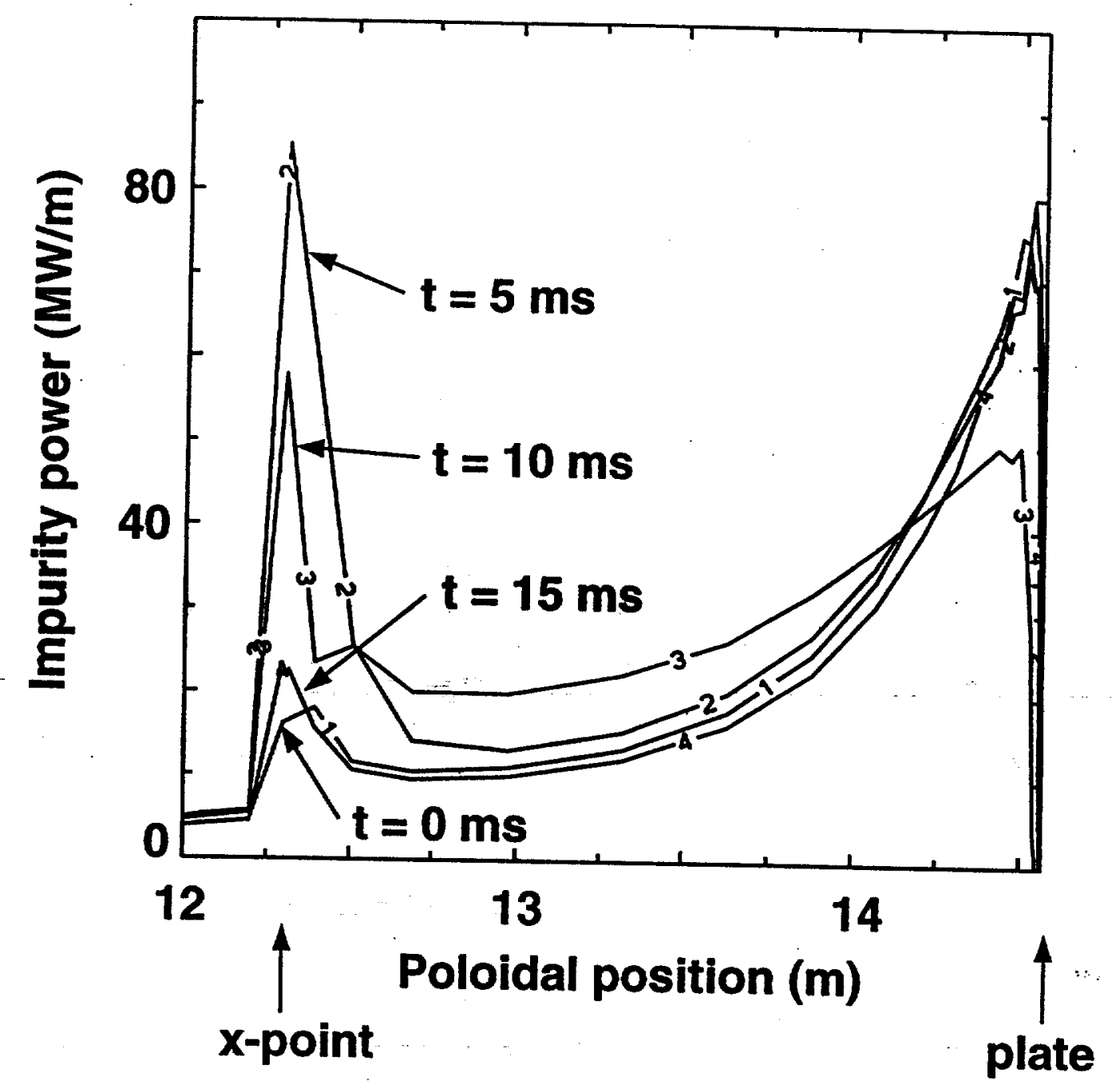

Figure 9 


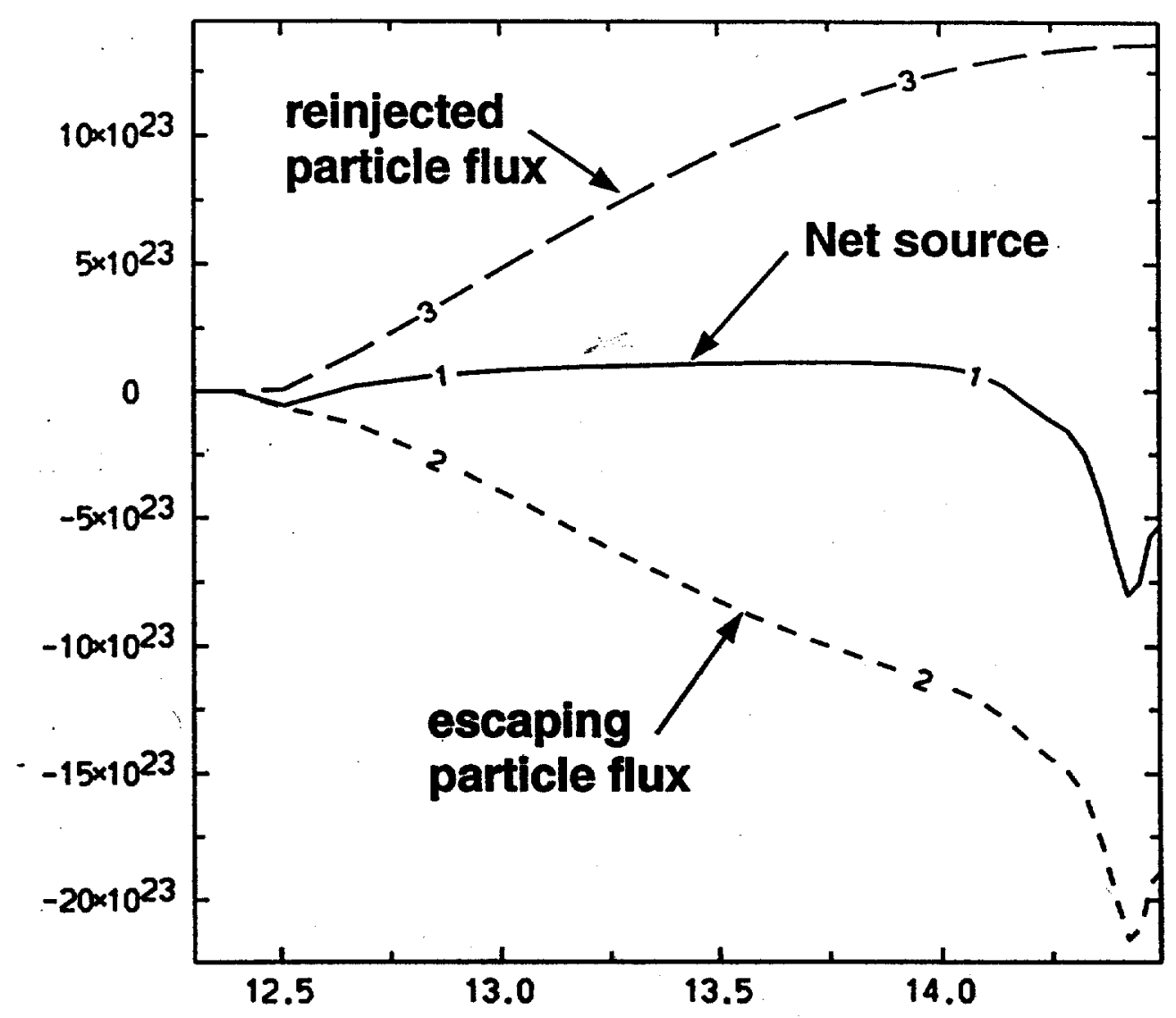

Figure 10 

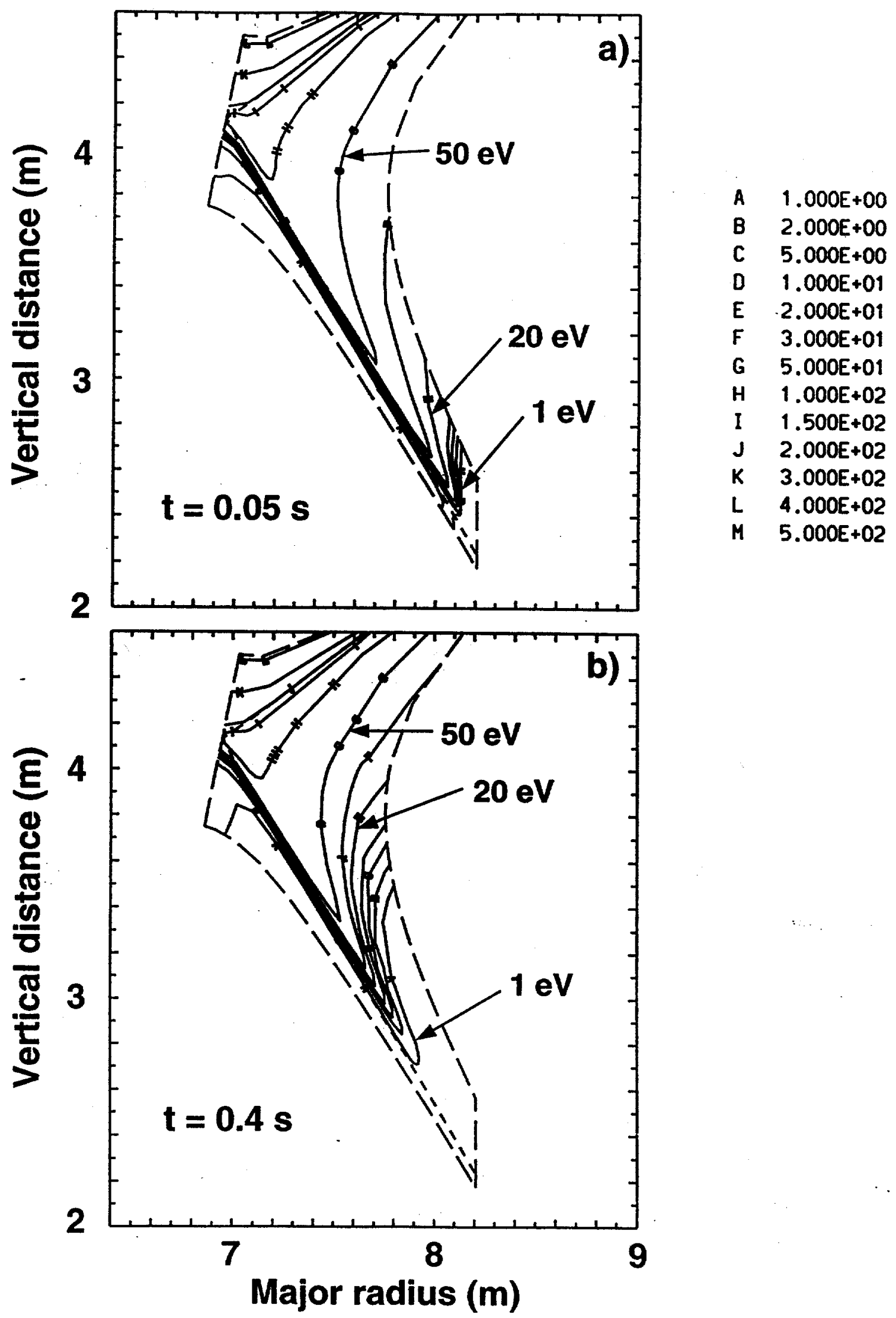

Figure 11 


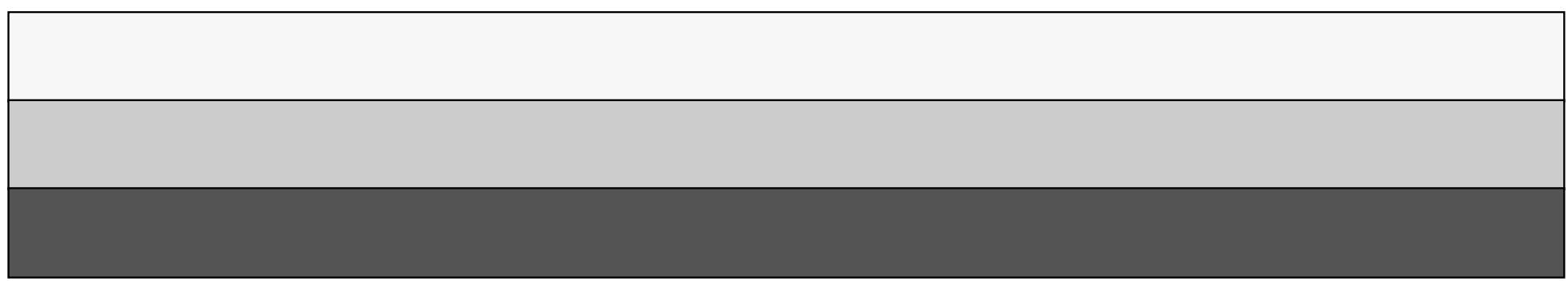

